\title{
碲参与的有机化学反应
}

\author{
陈 颖 $a, b$ 邓 金金 ${ }^{a, b}$ 景崤壁 $*, a$ 周宏伟 $*, b$ \\ ( $\left(^{a}\right.$ 扬州大学化学化工学院 江苏扬州 225002) \\ ( ${ }^{b}$ 嘉兴学院生物与化学工程学院 浙江嘉兴 314001)
}

\begin{abstract}
摘要 作为硫族元素的一员, 碲元素并没有像氧、硫、硒那样被人们所熟知. 它作为元素周期表中的 “隐形” 元素, 在 化学家们的视线中出现频率较低. 然而, 在过去的几十年里, 无论在材料科学、医学还是生物方面, 碲元素作为 “黑夜 中的翡翠”, 一直发挥着其独特的作用, 吸引越来越多的化学家前来驻足研究. 含碲化合物作为一种 “全新物质”, 在 有机化学方面所体现出来的性能无疑让化学家们眼前一亮. 近年来, 有关含碲化合物在有机化学方面的报道也越来越 多. 为了便于读者了解该领域的研究进展, 对其典型性工作进行了总结.
\end{abstract}

关键词 碲; 元素化学; 硫属; 绿色合成化学; 碲催化

\section{Tellurium-Mediated Organic Reactions}

\author{
Chen, Ying ${ }^{a, b}$ Deng, Xin ${ }^{a, b} \quad$ Jing, Xiaobi ${ }^{*, a} \quad$ Zhou, Hongwei*, \\ ( ${ }^{a}$ School of Chemistry and Chemical Engineering, Yangzhou University, Yangzhou, Jiangsu 225002) \\ $\left({ }^{b}\right.$ College of Biological, Chemical Sciences and Engineering, Jiaxing University, Jiaxing, Zhejiang 314001)
}

\begin{abstract}
As a member of chalcogen elements, tellurium is not as well known as oxygen, sulfur and selenium. As the "invisible" element in the periodic table of elements, it appears less frequently in the sight of chemists. However, in the past few decades, as the "emerald in the dark", tellurium has always played a unique role in the fields of materials science, medicine and biology, and attracted more and more chemists. In the eyes of some chemists, the performances of tellurium compounds in organic chemistry, as a "new substances", are undoubtedly eye-catching. Therefore, in recent years, there are increasing reports on the organic chemistry of tellurium compounds. In order to further understand these research progresses, the typical works are briefly summarized for readers' references.
\end{abstract}

Keywords tellurium; element chemistry; chalcogen; green synthetic chemistry; tellurium catalysis

碲元素是在 1782 年由特兰西瓦尼亚化学家弗朗茨约瑟夫•米勒・冯·赖兴施泰因在研究含金矿石时发现. 虽 然碲元素被发现的时间较早, 且在地壳中拥有相对来说 较丰富的含量(约百万分之 0.027 , 与银和金等元素相 当), 但它长期以来还是一种为人们所不熟知的元素, 在日常生活中的作用有限 ${ }^{[1]}$. 近年来, 由于人们对碲元 素的关注度越来越高. 其在材料、生物和医药等方面的 应用也越来越广泛. 例如, 二维碲纳米片可以作为一种 新型的光学材料, 被应用于非线性光学器件的制备. 经 碲纳米棒功能化处理后的钛涂层, 具有更强的抗菌性 能. 此外, 碲纳米线还可以作为无机纳米前药, 与细胞 中的 $\mathrm{H}_{2} \mathrm{O}_{2}$ 反应, 生成 $\mathrm{TeO}_{6}^{6-}$, 并可对肿瘤进行良好的
选择性治疗 ${ }^{[2]}$. 与硒元素类似 ${ }^{[3]}$, 由其优良的氧运载 ${ }^{[4]}$ 功 能所带来的氧化还原特性是碲元素最令人感兴趣的性 能之一, 同时这也是碲能参与许多有机反应的重要原因 之一 ${ }^{[5]}$. 不仅如此, 由于碲元素在元素周期表中位于第 $\mathrm{VI}$ 主族, 第五周期, 故其还具有一部分金属特性, 使得 含碲化合物能够催化有机反应 ${ }^{[6]}$, 且碲催化反应与硒催 化反应的机理有所不同 ${ }^{[7]}$. 利用碲元素与众不同的化学 特性, 有望开发出各种性能独特的新材料, 而碲参与的 有机化学反应, 是进行相关技术开发的基础. 因此, 本 文拟对该领域的几类典型反应进行归纳与总结, 希望能 够有助于研究者了解碲元素的一些基本反应性能, 从而 有利于后续相关技术的开发应用.

\footnotetext{
* Corresponding authors. E-mail: xbjing@yzu.edu.cn; zhouhw@zju.edu.cn Received May 10, 2020; revised June 3, 2020; published online July 8, 2020. Project supported by the Natural Science Foundation of Zhejiang Province (No. LY19B020004) and the Priority Academic Program Development of Jiangsu Higher Education Institutions. 浙江省自然科学基金(No. LY19B020004)及江苏省优势学科资助项目.
} 


\section{1 无机碲参与的有机反应}

碲单质作为最简单易得的碲源, 本身可以直接参与 有机化学反应. 与一些较活泼金属(如镁、锌)相似, 碲可 与卤化物反应. 1988 年, Amosova 等 $^{[8]}$ 开发了利用强碱性 还原体系 $\mathrm{KOH}-\mathrm{SnCl}_{2}-\mathrm{H}_{2} \mathrm{O}$ 制备烷基乙烯基碲化物 2 的 方法. 在该方法中, 碲单质、乙炔以及卤化物 1 直接反 应，直接合成烯基碲醚，从而实现了将碲元素引入有机 分子的合成目的(Scheme 1). 该方法以水为溶剂, 在 105 $115{ }^{\circ} \mathrm{C}$ 下反应 $5 \mathrm{~h}$, 即可以 $20 \% \sim 70 \%$ 的收率使底 物转化为目标产物. 与此同时, 还会产生少量的二乙烯 基碲化物 3 以及二烷基碲化物 4. 该方法中各产物的收 率与比例关系明显受到初始卤化物的种类与用量的影 响，其中，伯烷基卤化物与仲烷基卤化物反应效果明显， 而叔烷基乙烯基碲化物却难以获得, 主要是由于叔烷基 卤化物易在强碱性条件下脱去卤化氢而失去活性所导 致. 同时, 该方法的适用性按 $\mathrm{Br}>\mathrm{I}>\mathrm{Cl}$ 的顺序逐渐下 降. 随着卤化物用量的增加, 二乙烯基卤化物的产率也 会随之上升，几乎与烷基乙烯基碲化物产率一致. 虽然 该方法具有不少优点, 但过量 $\mathrm{KOH}$ 的使用以及 $\mathrm{Sn}$ 金属 的残留都会对环境造成一定的危害. 此外, 使用大量 $\mathrm{SnCl}_{2}$ 以及繁琐的反应操作都使得该方法在大规模合成 中的应用受到限制.

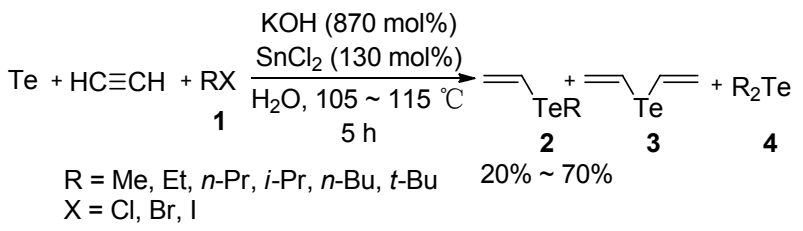

图式 1 烷基乙烯基碲化物的制备 Scheme 1 Preparation of alkyl vinyl telluride

碲单质还可以与金属有机化合物反应. 2000 年, Tanaka 等 ${ }^{[9]}$ 以有机膦作为催化剂催化碲单质插入 $\mathrm{Sn}$ $\mathrm{Sn}$ 和 $\mathrm{Pb}-\mathrm{Pb}$ 键, 从而构建新型含碲有机金属化合物. 有机锡化合物 5 与碲在温度为 $80{ }^{\circ} \mathrm{C}$, 苯为溶剂的情况 下反应，以较好的收率(34\% 100\%)得到含碲有机锡化 合物 6 (Scheme 2, a). 该方法不仅适用于芳香型有机锡 化合物，对烷基类有机锡化合物也有着较好的效果. 在 该反应中, 有机膦种类对产物的收率有着较大的影响. 例如: 三苯基膦催化三甲基二锡醚与碲单质反应时，只 能以 $45 \%$ 的收率得到相应的含碲化合物, 而同等条件下 三正丁基膦则能使产物的收率达到 100\% (Scheme 2, b). 而且该方法相较于直接使用有机碲膦化合物 $\left(\mathrm{R}_{3}{ }_{3} \mathrm{P}=\mathrm{Te}\right)$ 的方法而言, 效果相差不多. 例如: 三甲基二锡醚与三 叔丁基碲膦反应 $1 \mathrm{~h}$, 产物收率可以达到 $100 \%$. 与此同 时, 使用催化量的三叔丁基膦催化三甲基二锡醚与碲单
质反应 $3 \mathrm{~h}$ ，也能以 $100 \%$ 收率得到产物. 但该方法避免 了使用有机碲膦化合物而产生等物质的量的 $\mathrm{R}_{3}{ }_{3} \mathrm{P}$ 的问 题，从而减轻了底物纯化的困难. 不仅如此，该方法还 同时解决了有机碲膦化合物由于热不稳定性及高空气 敏感性而难以制备的问题，并可拓展到利用有机铅化合 物 7 来制备含碲有机铅化合物 8 的过程中(Scheme 2, c). 然而，使用高毒性的苯作为反应溶剂，使得该方法较易 对环境造成污染，同时也增加了后处理的成本.

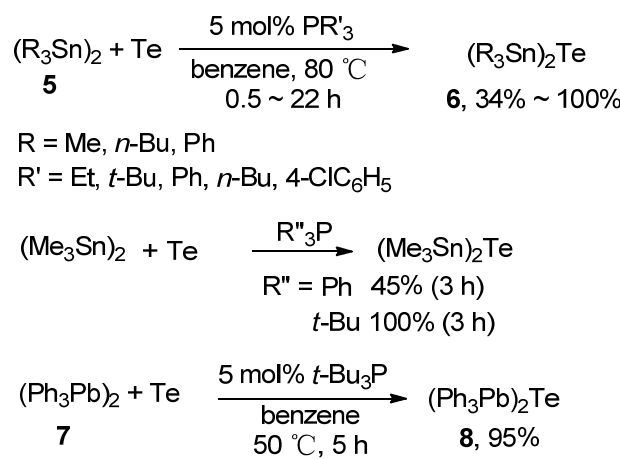

图式 2 含碲有机金属化合物的制备

Scheme 2 Preparation of organometallic compounds containing tellurium

从机理上来看，有机膦化合物与碲单质发生加成反 应, 得到相应的有机碲膦化合物 9, 接着再与有机锡化 合物反应，生成含碲有机锡化合物 10, 同时生成的有机 膦化合物进入另一个催化循环体系继续参与反应. 该方 法实质上还是以有机碲膦化合物作为碲元素的转移基 质，但只需催化量的有机膦化合物就可以完成反应. 同 时也为后续的底物纯化带来了较大的便利(Scheme 3).

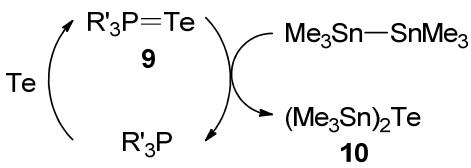

图式 3 有机膦催化的 $\mathrm{Te}$ 插入 $\mathrm{Sn}-\mathrm{Sn}$ 键的可能机理 Scheme 3 Possible mechanism for the organophosphine-catalyzed insertion of tellurium into $\mathrm{Sn}-\mathrm{Sn}$ bond

有机非金属盐也可与碲单质反应，合成含碲化合 物. 2019 年，谭启涛等 ${ }^{[10]}$ 利用碲粉与易得的二芳基碘鎓 盐 11 合成了嵌入碲的大环芳香族化合物 12 (Scheme 4). 该方法使用 2-甲基吡啶作为添加剂，二甲基亚砜 (DMSO) 作为溶剂来进行反应, 能够以较好的收率 $(35 \% \sim 81 \%)$ 得到目标产物. 其中，单取代的二芳基碘 鎓盐和 3,7-二取代的二芳基碘鎓盐，无论取代基团的电 子效应如何，其都能以良好的效率得到目标化合物. 与 此同时，合成的含碲芳香族化合物表现出良好的空气/ 水分稳定性和高热稳定性，故无需采取特殊的保护措 
施，即可通过柱层析分离得到目标产物. 令人惊讶的是， 该方法对含喹啉结构的二芳基碘鎓盐也可以起到相对 良好的效果. 而且在含有生物活性的类黄酮中也能成功 地引入碲(图 1), 这对于研究含碲化合物潜在的生物性 能起到了一定的作用. 该方法还可以拓展到许多其他含 碲共轭体系的杂环合成中. 不仅如此, 合成的固态物质 还具有有趣的室温可控磷光效应, 这也为新型室温可控 磷光效应材料的研究提供了参考.

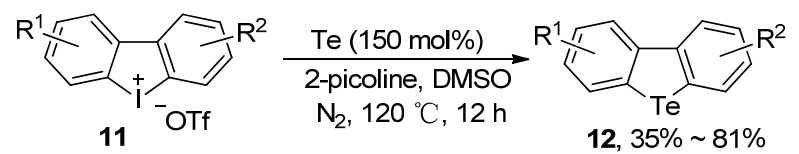

$\mathrm{R}^{1}, \mathrm{R}^{2}=\mathrm{Me}, \mathrm{CF}_{3}, \mathrm{Br}, \mathrm{CO}_{2} \mathrm{Me}$, isoquinoline, benzo- $\gamma$-pyrone

图式 4 含碲大环芳香族化合物的制备

Scheme 4 Preparation of large cyclic aromatic compounds containing tellurium
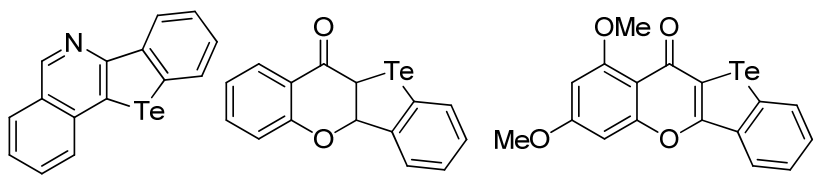

图 1 含喹啉、类黄酮结构的代表性化合物

Figure 1 Representative compounds containing quinoline and flavonoid structures

\section{2 无机碲催化的有机反应}

碲的金属性使其能够如金属一样催化有机化学反 应. 与一些贵金属催化剂(如钯、钉等)相似，碲可催化插 羰反应. Sonoda 等 ${ }^{[5 b]}$ 发现, 在一氧化碳氛围中, 碲单质 在催化胺类化合物 13 转化为尿素衍生物 14 以及甲酰胺 类化合物 15 方面能够起到一定的作用(Scheme 5). 虽然 两种产物的收率较低, 但反应体系干净, 副产物仅为氢 气. 而氢气能够作为清洁能源被广泛应用, 所以该反应 还是比较环保的. 此外, 该反应的收率受反应时间的影 响比较明显. $100 \mathrm{mmol}$ 的正叔丁基胺在 $1 \mathrm{~mol} \%$ 碲催化 下反应 $10 \mathrm{~h}$, 能够得到 $7.8 \mathrm{~mol}$ 的 $N$-二正丁基尿素, 7.2 $\mathrm{mmol}$ 的氢气以及 $17.3 \mathrm{mmol}$ 的 $N$-正丁基甲酰胺. 而延 长反应时间至 $72 \mathrm{~h}$, 三种产物的产量均能够达到原来的 3 倍左右. 与此同时, 提升反应温度以及增加一氧化碳 压力都能使得三种产物的收率升高, 其中较高的反应温 度以及较低的一氧化碳压力对于产物中尿素衍生物含 量占比的提升有益. 而选用硒来代替碲催化反应时, 尿 素衍生物的产率就会明显下降, 可见在该方法中碲拥有 比硒更好的催化活性. 该方法对于脂肪族胺类化合物和 芳香族胺类化合物均适用, 且两者的反应效果相差不 大.

令人惊喜的是，当向反应体系中添加 $10 \mathrm{~mol} \%$ 的硝

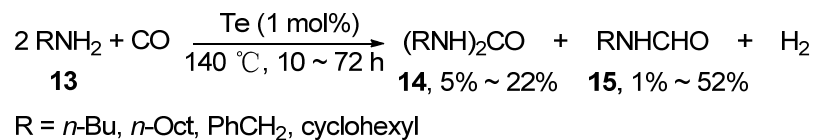

图式 5 碲催化胺类化合物的酰基化反应

Scheme 5 Tellurium catalyzed acylation of amine compounds

基苯时，能够显著地抑制氢气以及甲酰胺类化合物的生 成，同时不会对尿素的收率造成影响. 从机理上可知, 碲在催化胺类化合物与一氧化碳反应后变成碲化氢 16, 而此时硝基苯利用其氧化性，将碲化氢氧化为碲单质， 自身变成苯胺(可被分离得到). 而反应中使用硝基苯作 为添加剂能够抑制甲酰胺类化合物的生成，也说明了碲 化氢在甲酰胺类化合物的产生过程中起到了重要的作 用(Scheme 6).

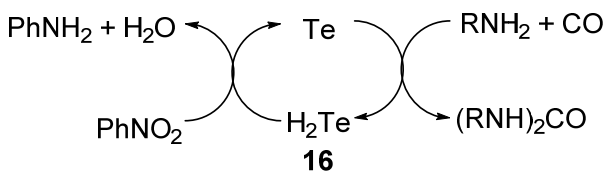

图式 6 硝基苯存在下 $\mathrm{Te}$ 催化胺与一氧化碳炭基化反应的机 理

Scheme 6 Mechanism of Te-catalyzed carbonylation of amine with carbon monoxide in the presence of nitrobenzene

一系列控制实验结果表明，该方法中碲作为催化剂 先催化胺类化合物和一氧化碳反应，自身被还原为碲化 氢 16. 接着 $\mathrm{H}_{2} \mathrm{Te}$ 脱氢分解为 $\mathrm{H}_{2}$ 与碲单质. 而碲单质作 为催化剂继续参与下一个循环. 另一方面, 一氧化碳与 碲化氢发生插入反应得到碲代甲酸 17 , 碲代甲酸与胺 类化合物反应得到甲酰胺类化合物与 $\mathrm{H}_{2} \mathrm{Te}$. 而碲化氢 则继续参与反应(Scheme 7).

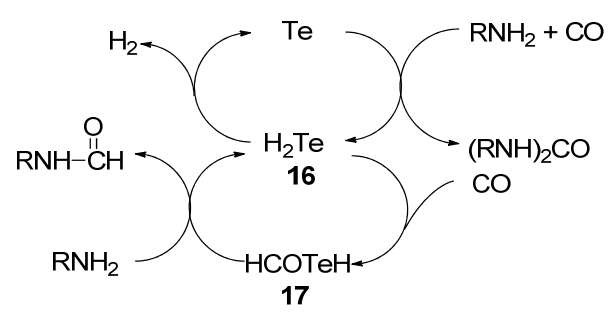

图式 7 碲催化胺与一氧化碳羰基化反应的可能机理 Scheme 7 Possible mechanism of Te-catalyzed carbonylation of amines with carbon monoxide

与金属氧化物类似，二氧化碲可催化各种 $\mathrm{C}-\mathrm{H}$ 键 的氧化反应，从而实现其官能化. Bergman 等 ${ }^{[1]}$ 发现, 在含 $\mathrm{LiBr}$ 的醋酸溶液中, 二氧化碲能够将溶液中的乙 酸缓慢氧化成乙酰氧基卡宾, 并与芳香族化合物 18 反 应，最终得到乙酰氧基甲基化产物 19 (Scheme 8). 该方 法虽然耗时长, 产率较低(5\% 30\%). 但反应体系干净, 副产物二芳基甲烷衍生物较少, 且没有出现溴化产物. 
例如: 对二甲苯在该方法下能以 $30 \%$ 的收率得到乙酸2,5-二甲基苄酯, 并且没有副产物的产生. 而在类似的 反应环境下, 利用碲酸氧化甲苯衍生物 20 可以得到甲 苯衍生物侧链乙酰氧基化的产物 $\mathbf{2 1}$, 且大幅度缩减了 反应的时间(Scheme 9). 但同时也产生了苯甲酫以及芳 环溴化的副产物, 这使得反应的后处理变得更为繁琐。 例如: 邻二甲苯在此方法下能够以 $48 \%$ 的收率得乙酸2-甲基苄酯，同时会产生少量的二甲基苯甲醛以及 2,5二甲基溴苯. 此外, 对于苯环电子云密度较高的芳香族 化合物, 芳环的溴化会成为主要反应, 例如 4-甲氧基甲 苯在此条件下反应时以 $68 \%$ 的收率得到 2-溴-4-甲氧基 甲苯. 该方法也体现了含碲化物在 $\mathrm{C}-\mathrm{H}$ 键活化方面的 有着一定的作用, 同时也展现了其优异的氧化性能, 但 较高的反应温度与复杂的反应体系使得反应适用性受 到影响.

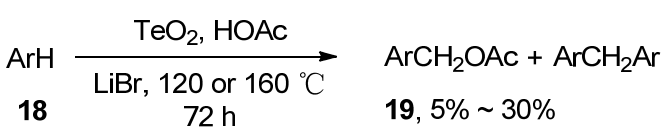

图式 8 二氧化碲催化的芳香族化合物的乙酰氧基甲基化反 应

Scheme 8 Acetyloxymethylation of aromatic compounds catalyzed by tellurium dioxide

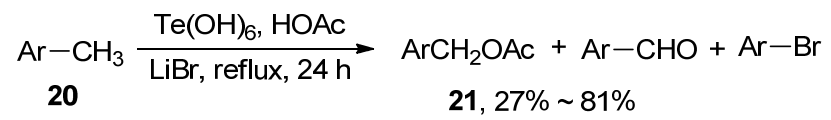

图式 9 碲酸催化的甲苯侧链的乙酰氧基化反应

Scheme 9 Acetylation of toluene side chains catalyzed by telluric acid

一系列控制实验结果表明, $\mathrm{TeO}_{2}$ 先与溶液中的醋酸 和溴化锂反应得到中间体 $\mathbf{2 2}$, 然后中间体 $\mathbf{2 2}$ 变成其共 振中间体 23, 中间体 23 在醋酸根离子的进攻下变成中 间体 24, 中间体 24 易发生解离得到二价碲化合物以及 其他化合物. 同时, 中间体 $\mathbf{2 4}$ 在醋酸根离子的作用下解 离成二价碲化合物及乙酰氧基甲基卡宾. 之后, 乙酰氧 基甲基卡宾进攻芳香族化合物得到最终产物乙酰氧基 甲基化芳香族化合物(Scheme 10).

甲苯侧链乙酰氧基化反应中, $\mathrm{Te}(\mathrm{OH})_{6}$ 先将溶液中 的溴离子氧化成溴单质, 而自身被还原为四价碲化合 物. 然后甲苯与溴单质反应得到溴代甲苯与溴化氢. 其 中, 溴代甲苯在四价碲化合物以及醋酸的作用下变成乙 酰化甲苯与溴化氢, 溴化氢则继续参与反应循环 (Scheme 11).

除氧化反应外，碲还可以催化硫化反应.Fujiwan 等 ${ }^{[12]}$ 发现, 碲在由异氧化物 25 和硫单质合成异硫氧酸

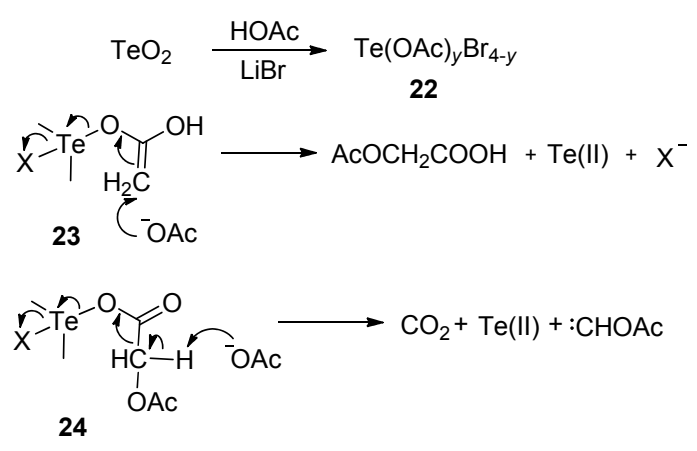

图式 10 芳香族化合物乙酰氧基甲基化反应的可能机理 Scheme 10 Possible mechanisms of acetyloxymethylation of aromatic compounds

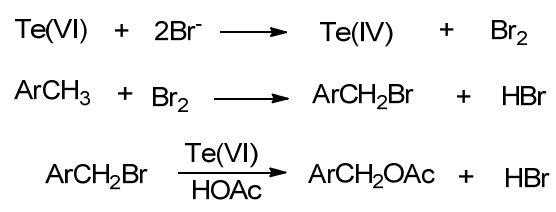

图式 11 甲苯侧链乙酰氧基化反应的可能机理 Scheme 11 Possible mechanisms of acetylation of toluene side chains

酯 26 的过程中有着较好的催化作用(Scheme 12). 该反 应中异氰化物与硫单质在三乙胺为添加剂, 四氢呋喃为 溶剂的情况下能够以良好的收率 $(69 \% \sim 85 \%)$ 得到异硫 氧酸酯. 该方法不仅适用于芳香族化合物，对于脂肪族 异氧化物也有着一定的作用. 例如: 茮基异氧能够在该 方法下以 $79 \%$ 的收率得到芐基异硫氰酸酯, 而叔丁基异 氧也能够以 $69 \%$ 的中等收率得到叔丁基异硫氰酸酯. 可 见该方法适用性较广. 不仅如此, 该反应中所需催化剂 碲用量较少, 而且同等条件下碲的催化能力远高于硒的 催化能力. 例如: 碲与硒用量均为 $0.5 \mathrm{~mol} \%$, 且其他条 件都一样的情况下, 碲催化反应并使得产物收率达到 $100 \%$ 所用的时间为 $3 \mathrm{~h}$, 而相同时间内硒催化反应得到 的产物收率不足 $40 \%$ (图 2). 由于该反应的原子经济性 较高, 避免了许多废弃物的产生, 所以反应的后处理也 更为简便. 但过量刺激性三乙胺的使用, 使得操作过程 有潜在风险，降低了反应的安全性.

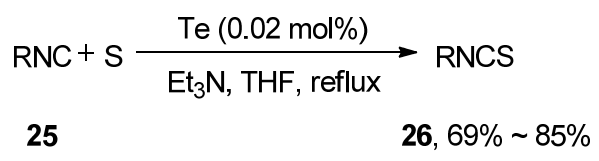

$\mathrm{R}=\mathrm{Me}, n-\mathrm{Bu}, t-\mathrm{Bu}, \mathrm{PhCH}_{2}$, cyclohexyl

图式 12 碲单质催化的异硫氰酸酯的合成 Scheme 12 Synthesis of isothiocyanates catalyzed by tellurium elements

\section{3 有机碲参与的有机反应}

与无机碲相比, 有机碲化合物结构更加复杂, 从而 有着更加多样化的反应活性. 有机碲参与的反应被广泛 


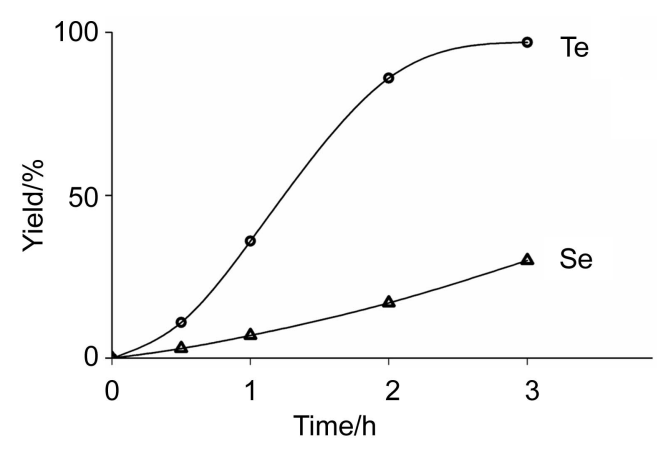

图 2 碲与硒催化性能的比较

Figure 2 Comparison of catalytic properties of tellurium and selenium

应用于含碲化合物的合成. 1989 年, Comasseto 课题组 ${ }^{[13]}$ 利用含双键结构的端位炔烃 27 和有机碲化合物 $\mathbf{2 8}$ 反应 得到全新的含碲烯烃化合物 29 和 30 (Scheme 13, a). 对 于脂肪族炔烃和芳香族炔烃而言, 该反应的结果在产物 的比例关系方面存在着一定的区别. 其中, 有机碲化合 物与芳香族炔烃反应, 单一地得到反马氏加成的含碲烯 烃化合物 29. 有机碲化合物与脂肪族炔烃反应, 除反马 氏加成的含碲烯烃主产物外, 还会有部分马氏加成的产 物 30 生成. 除较好的区域选择性外, 反应也具有较好的 立体选择性, 所有产物中 $Z$ 式构型的产物占比都超过 $E$ 式构型的产物, 比例范围为 $2.57 \sim 8.09: 1$. 不仅如此, 对于脂肪族和芳香族有机碲化合物而言, 该方法都具有 良好的适用性. 例如: (2Z)-3-甲基-2-烯-4-炔-1-戊醇与二 十二烷基二碲醚反应，能以 $80 \%$ 的收率得到相应结构的 化合物 29. 在此基础上, 还利用二乙烯基有机碲化合物 32 与苯乙炔衍生物 31 制备了全新的不对称二乙烯基有 机碲化合物 33 (Scheme 13, b). 和之前端位炔烃与有机 碲化合物反应的结果类似, 该方法得到的主要产物依旧 是 $Z$ 式构型的反马氏加成产物. 此外还有少量的 $E$ 式构 型的反马氏加成产物. 例如: 苯乙炔与二乙烯基二碲醚 反应，能以 $76 \%$ 的收率得到相应结构的化合物 33. 可见 该方法适用性较广，而且立体选择性良好. 但 $2.5 \sim 5.0$ equiv. 炔烃的使用使得反应的原子经济性大大降低, 且 易燃品 $\mathrm{NaBH}_{4}$ 的使用, 也会导致反应的安全系数受到 影响.

含碲化学键较弱, 容易发生均裂而产生碲自由基. 该反应在可见光驱动下即可发生. 例如, Sonoda 等 ${ }^{[14]}$ 发 现, 将二苯基二碲醚 $\mathbf{3 5}$ 加入炔烃衍生物 $\mathbf{3 4}$ 中, 并在无 溶剂条件下用光照射一段时间, 能够产生 $E$ 式构型为主 的含碲烯烃类化合物 36 (Scheme 14). 此外炔烃中的 R 基团会对产物的收率造成较大的影响. 当炔烃衍生物为 脂肪族炔烃时, 由于产物在近紫外有吸收, 所以容易导 致产物发生逆反应, 分解为炔烃衍生物和二苯基二碲 醚. 例如：1-辛炔在波长为 $300 \mathrm{~nm}$ 的光照射下, 仅有

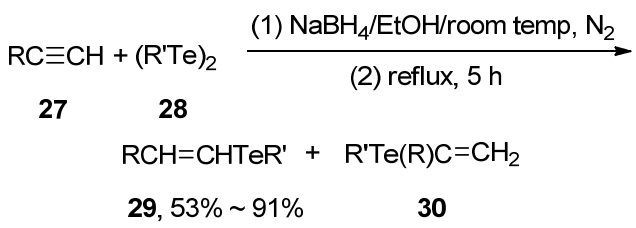

33, $36 \% \sim 93 \%$

图式 13 含碲烯烃化合物的制备

Scheme 13 Preparation of tellurium containing olefin compounds

43\%的核磁产率. 而添加偏正光片过滤波长为 300 400 $\mathrm{nm}$ 的光之后，产物的核磁收率为 $62 \%$, 而分离收率则 能够达到 $29 \%$. 而当炔烃上连有环己烯基等与参键共轭 的官能团时，则能够使化合物的吸收峰偏移，从而避免 上述问题. 例如丁炔二酸二乙酯和二苯基二碲醚反应, 能以 $90 \%$ 的收率得到加成产物. 并且仅需用波长为 300 $\mathrm{nm}$ 的光来照射反应体系即可. 该方法中产物选择性良 好，而且反应体系干净. 不仅如此，该方法也充分体现 了有机碲化合物具有光敏性的特点, 为后续研究有机碲 化合物的光学性能提供了参考.

$$
\begin{aligned}
& \left.\mathrm{R}=+(\mathrm{PhTe})_{2} \frac{\mathrm{hv}(\geq 300 \mathrm{~nm} \text { or } \geq 400 \mathrm{~nm})}{40 \sim 70^{\circ} \mathrm{C}, 20 \sim 96 \mathrm{~h}} \underset{\mathrm{R}}{\stackrel{\mathrm{PhTe}}{=}}\right\rangle_{\mathrm{TePh}} \\
& 34 \quad 35 \\
& \text { 36, } 29 \% \sim 90 \%
\end{aligned}
$$

$\mathrm{R}=n$-Hex, $\mathrm{Ph}, \mathrm{HOMe}, \mathrm{H}_{2} \mathrm{NMe}, \mathrm{CHO}, \mathrm{CO}_{2} \mathrm{Et}$, cyclohexenyl, isopropenyl

图式 14 二苯基二碲醚与炔烃化合物的光化学反应 Scheme 14 Photochemical reaction of diphenyl ditelluride with alkynes

除了光照外，一些过渡金属催化剂(如铜)也可促进 碲键断裂. 2006 年, Sangit 等 ${ }^{[15]}$ 在 $\mathrm{CuI} / \mathrm{Bpy}$ 与过量镁粉的 存在下, 利用微波加热使二苯基二碲醚 35 与卤代芳基 37 发生反应，从而得到相应的单碲醚产物 38 (Scheme 15). 该方法大大缩减了利用卤代芳基和二苯基二碲醚 来制备单碲醚的时间. 除此以外，该反应中无论是含杂 环的卤代芳基还是大位阻的卤代芳基，都能表现出良好 的官能团容忍性. 例如，二苯基二碲醚与 1-溴碘荎反应， 以 $68 \%$ 的收率得到相应的单碲醚. 而含有杂环的 3-溴吡 啶在该方法中以 $62 \%$ 的收率得到相应的单碲醚. 此外, 卤素的种类也会对产物的收率产生一定的影响.二苯基 二碲醚与溴苯反应产率最高，为 $65 \%$; 与碘苯反应的效 果就稍差一点，产物收率仅有 $60 \%$; 而与氯苯的反应效 果最差, 在反应升温到 $200{ }^{\circ} \mathrm{C}$ 时, 产物收率依旧不太理 想，仅有 $40 \%$. 该反应可以用于制备不同的含碲抗氧化 剂(图 3), 且对于单硫醚和单硒醚的制备也有着一定的 
作用. 但是, 该反应也有着比较明显的缺陷, 较高的反 应温度以及过量镁粉的使用都会使得该反应在应用方 面受到较大的限制.

$$
\begin{array}{cccc}
\mathrm{Ar}-\mathrm{X} & + & \mathrm{Cul} / \text { bipyridyl } & \\
37 & 35 & \mathrm{PhTe})_{2} & \mathrm{Ar}-\mathrm{Te}-\mathrm{Ph} \\
\mathrm{X}=\mathrm{Br}, \mathrm{I}, \mathrm{Cl} & 200^{\circ} \mathrm{C}, 6 \mathrm{~h} & 38
\end{array}
$$

图式 15 卤代芳烃与二碲醚的反应

Scheme 15 Reaction of halogenated aromatic hydrocarbons with tellurium ethers<smiles>CC1=CC(C)(C)Nc2ccc(-c3ccccc3)cc21</smiles><smiles>COc1ccc(O)c([Te]c2ccccc2)n1</smiles>

图 3 卤代芳烃与二碲醚反应制备的含碲抗氧化剂 Figure 3 Tellurium-containing antioxidant prepared by reaction of halogenated aromatic hydrocarbon and ditellurium ether

\section{4 有机碲催化的有机反应}

有机碲化合物的高反应活性使其在一些反应中亦 可以充当催化剂. 2001 年, Detty 等 ${ }^{[16]}$ 利用有机碲化合物 催化烯烃的碘化 (Scheme 16). 该方法利用无机碘化物作 为硒源，让底物 39 在磷酸盐缓冲溶液中进行反应，以中 等至良好的收率(40\% 94\%)得到碘代产物 40. 该反应 通过有机碲化物对双氧水进行活化, 从而氧化无机碘化 物并对底物进行碘化反应. 从反应机理上可知，首先无 机碘化物与催化剂在双氧水的作用下发生加成反应，同 时催化剂上的氧原子对碲进行鳌合配位，防止另一个碘 化物分子加成到碲原子上, 并最终形成中间体 41(图 4). 而中间体 41 的正电荷则激活碘配体, 从而完成对底物 的亲核进攻(Scheme 17). 而且使用 $\mathrm{pH}$ 为 6 的磷酸盐缓 冲溶液作为溶剂也增加了酸性底物对反应体系的耐受 性. 不仅如此, 若底物具有一定的水溶性, 则可在不使 用有机助溶剂的情形下进行反应. 例如: 4-戊烯酸钠在 不添加醚作为有机助溶剂的情况下，即可以 $88 \%$ 的收率 得到碘代产物. 而且该方法与直接使用碘单质来进行碘 化反应得到结果几乎一致, 其中大部分底物的收率都有 略微提升. 但是对于一些未被活化的底物(例如苯和甲 苯)而言, 该催化剂催化的效果就显得相对较差. 进一 步研究表明该方法对于利用无机溴化物来对底物进行 溴化的效果并不突出, 所以其适用范围存在一定的限 制. 但该方法依旧具有很多优点, 例如: 在易于底物纯 化的同时，也尽可能地避免了有机卤化物对环境的污 染. 而且此反应对底物的兼容性较好, 产物收率受底物 空间位阻效应的影响也较小. 但是过量强氧化剂的使用 也对该反应的安全性造成了不可忽视的影响, 从而使得
该方法在应用方面受到限制.

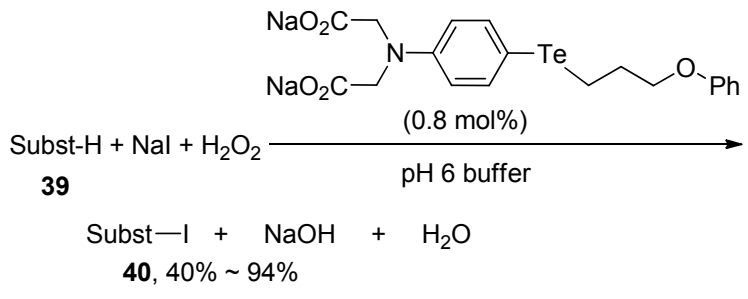

Subst $-\mathrm{H}=$ mesitylene, 4-pentenoic acid, 4-penten-1-ol, $N, N$-dimethylaniline

图式 16 有机碲催化的碘化反应

Scheme 16 Iodination reaction catalyzed by organotellurium

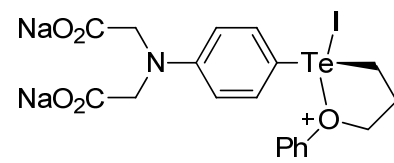

39

图 4 碘化反应的中间体

Figure 4 Intermediate of an iodized reaction

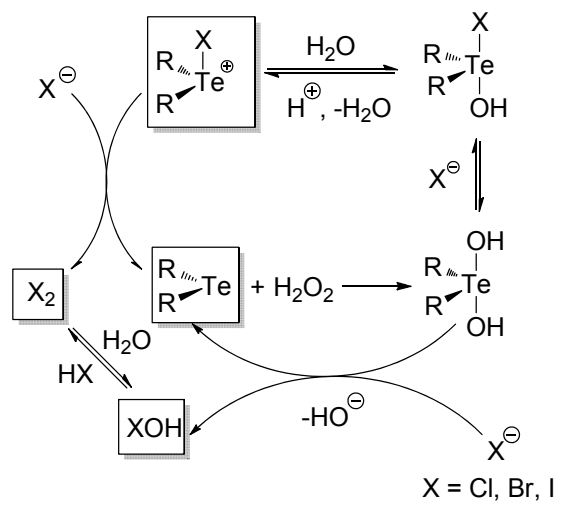

图式 17 有机碲的循环机理

Scheme 17 Cycle mechanism of organic tellurium

除卤化反应外，碲化合物还可以催化氧化反应. 在 这些反应中，碲作为氧转移催化剂，起到运载氧的效果. 2005 年, Parvez 等 ${ }^{[17]}$ 利用环状亚碲酸酯和螺二氧基碲烷 等有机碲化合物催化硫酚 42 与过氧化物 $\mathbf{4 3}$ 反应，得到 相应的二硫化物 44 (Scheme 18). 该工作利用半衰期 $\left(t_{1 / 2}\right)$ (将一半硫醇氧化成二硫醚所用时间)来表征各催化 剂的催化活性. 其中, 环状亚碲酸酯催化性能较环状亚 硒酸酯而言显得比较突出. 当使用 $56 \%$ 过氧化叔丁醇作 为过氧化物模拟物时, 环状亚硒酸酯催化底物所需半衰 期为 $90 \mathrm{~h}$, 而环状亚碲酸酯催化底物所需半衰期仅有 $0.05 \mathrm{~h}$ ，可以明显地看出环状亚碲酸酯的催化性能高于 环状亚硒酸酯的催化性能. 而且环状亚硒酸酯对于过氧 化物种类以及浓度依赖过高. 当过氧化叔丁醇用量为 $90 \%$ 时，环状亚硒酸酯催化底物所需半衰期为 $2.5 \mathrm{~h}$, 而 过氧化叔丁醇用量下降至 $56 \%$ 时, 所需半衰期骤增到 
$90 \mathrm{~h}$. 除了环状亚碲酸酯以外, 螺二氧基碲烷也展现出 很好的催化能力, 其催化效果与环状亚碲酸酯的催化效 果相近. 而且此类化合物作为硒代谷胱甘肽过氧化物酶 的模拟物, 还能够有效地减少有害的过氧化物来保护细 胞免受氧化应激. 所以有机碲化合物除了有较好的催化 活性之外，还有一定的生物活性.

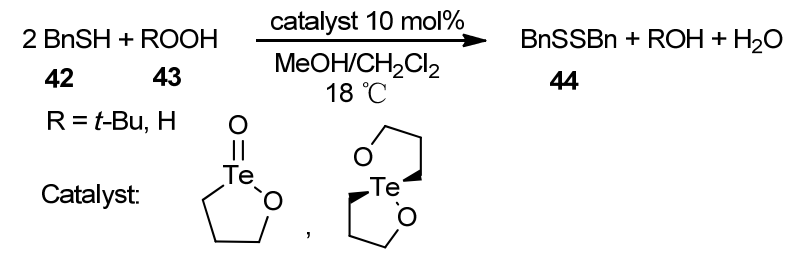

图式 18 有机碲催化硫酚与过氧化物的反应

Scheme 18 Organic tellurium catalyzed reaction of thiophenol with peroxides

肟类是易得的有机化合物，而以肜为原料的反应， 有着很好的应用价值 ${ }^{[18]}$. 其中, 脱肜反应被广泛应用于 天然产物合成与药物合成中 ${ }^{[19]}$. 受上述碲催化氧化反 应的启发, 我们 ${ }^{[20]}$ 最近以二苯基二碲醚作为催化剂来 催化氧化脱肜反应(Scheme 19). 该反应可在无溶剂条 件下进行, 并使用氧气作为氧化剂. 只需要使用 2.5 $\mathrm{mol} \%$ 二苯基二碲醚作催化剂, 即可使得大部分肟(45)在 $60{ }^{\circ} \mathrm{C}$ 下以 $54 \% \sim 94 \%$ 的收率转化为酮 (46). 当芳环上连 有给电子基团时, 产物收率会比连有吸电子基团的产物 收率高. 而且底物的位阻越大, 对底物收率造成的影响 也就越大，需要更高的温度才能完成反应. 例如：苯丁 酮肜在参与反应时, 在 $60{ }^{\circ} \mathrm{C}$ 下就能以 $54 \%$ 的收率得到 苯丁酮, 而苯戊酮肜在 $60{ }^{\circ} \mathrm{C}$ 时却难以发生反应, 需要 将反应温度升至 $80{ }^{\circ} \mathrm{C}$ 才能得到苯戊酮. 不仅如此, 该 方法对脂肪族酮肟以及杂环酮肟也适用。

通过机理研究, 可推测出如下反应机理: $(\mathrm{PhTe})_{2}$ (35)首先被氧化成次碲酸酐即 PhTeOTePh (47), 随后裂 解成中间体 48 与 49 , 中间体 48 可以被氧化成中间体 49 , 而中间体 49 会进一步被氧气氧化成中间体 $\mathbf{5 0}$, 中间体 50 与肜 45 加成得到中间体 $\mathbf{5 1}$, 这一步反应受底物的空 间位阻影响较大, 有时需要更高的反应温度才能实现, 中间体 51 可分解为主要产物酮、副产物 HNO 以及中间 体 49, 而其中产生的中间体 49 则作为催化物质重新启 动催化循环圈 $\mathrm{A}$. 中间体 49 也可能直接与肪 $\mathbf{4 5}$ 加成得 到中间体 52, 并分解为副产物 $\mathrm{HNO}$ 、主要产物酮以及

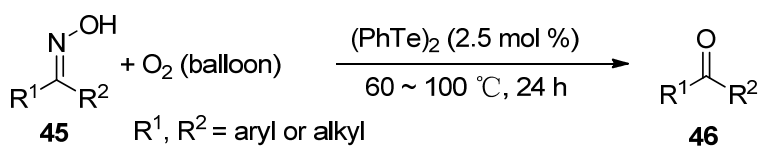

图式 19 有机碲催化的脱肜反应

Scheme 19 Deoxime reaction catalyzed by organic tellurium
中间体 48. 中间体 48 会作为另一个催化物种重新启动 催化循环圈 B. 该方法经历了一个自由基反应的历程, 从而与主要以离子型机理进行的有机硒催化氧化脱肜 反应大不相同(Scheme 20) $)^{[21]}$.

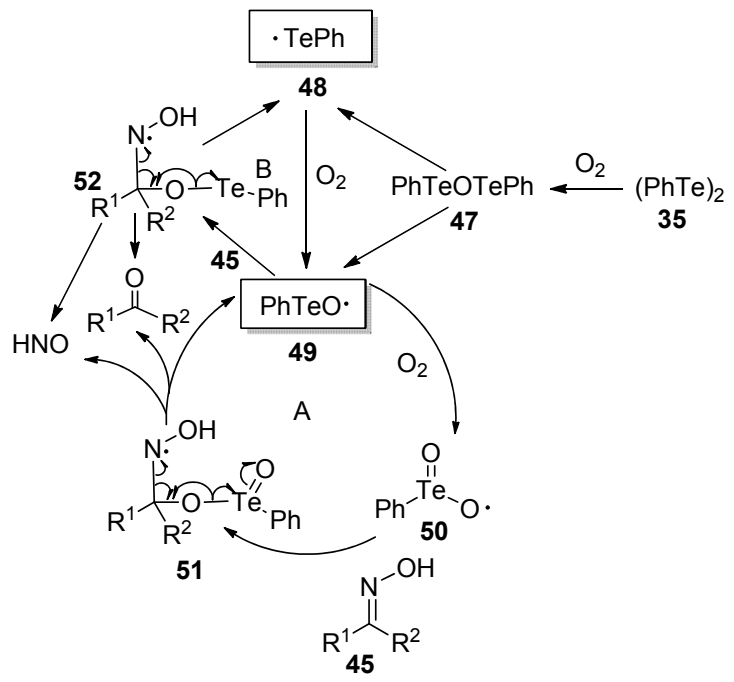

图式 20 二苯基二碲醚催化脱肟反应的可能机理 Scheme 20 Possible mechanism of the $(\mathrm{PhTe})_{2}$-catalyzed deoximation reaction

\section{5 结论}

由上述可知，碲元素独特的反应活性源自于其准金 属特性.一方面，碲作为非金属元素，易于形成各种共 价键，从而便于与有机化合物反应; 另一方面，碲元素 的金属性使其能够表现出一些金属特性，例如，易与卤 代烃反应生成含碲试剂, 易与一氧化碳发生作用从而可 催化插羰反应，易被氧化还原从而可催化氧化反应。与 同族的硒元素相比，含碲化学键更弱，从而易发生均裂 反应，产生自由基. 因此，碲催化反应可通过自由基机 理进行, 而与硒催化反应不同. 与无机碲相比, 有机碲 试剂结构更加复杂，也可通过引入不同取代基来调控其 反应性，从而使其应用范围更加广阔. 碲是较少见的准 金属元素，但其参与或催化的部分有机反应，已经表现 出一些绿色化学的特征, 符合有机合成化学的发展趋势 ${ }^{[22]}$. 碲虽然比硒贵 (约 8 元 $/ \mathrm{g}$ ), 但与传统的贵金属如铂 ${ }^{[23]}$ (约 180 元 $/ g$ )、钯 ${ }^{[24]}$ (约 500 元 $/ g$ )、钓 ${ }^{[25]}$ (约 70 元 $/ g$ )、金 ${ }^{[26]}$ (约 360 元 $/ g$ ) 等相比, 价格更加便宜. 因此, 碲 催化同样有望被应用于工业合成领域. 目前，人们对于 这方面的研究较少, 而该领域有待进一步深入研究.

\section{References}

[1] (a) Gelbstein, Y.; Rosenberg, Y.; Sadia, Y.; Dariel, M. P. J. Phys. Chem. C 2010, 114, 13126

(b) Cao, W.; Gu, Y.; Meineck, M.; Li, T.; Xu, H. J. Am. Chem. Soc. 2014, 136, 5132 . 
(c) Kaur, M.; Rob, A.; Williams, J. C.; Huang, Z. ACS Sym. Ser. 2013, 1152, 89.

[2] (a) Wu, L.; Huang, W.; Wang, Y.; Zhao, J.; Ma, D.; Xiang, Y.; Li, J.; Ponraj, J.; Dhanabalan, S.; Zhang, H. Adv. Funct. Mater. 2019, 29, 1806346.

(b) Park, D.; Gautam, M.; Park, M.; Hwang, J.; Yong, C.; Kim, J.; Byeon, J. Environ. Sci.-Nano. 2019, 6, 2074.

(c) David, M.; María, U.; William, T.; Marcial, F.; Ada, V.; Iván, M.; Yves, H.; Thomas, J.; José, M. Nanomed-Nanotechnol 2019, $17,36$.

(d) Wu, Y.; Guo, T.; Qiu, Y.; Liu, Y.; Yao, Y.; Lian, W.; Lin, L.; Song, J.; Yang, H. Chem. Sci. 2019, 10, 7068.

[3] (a) Cao, H.; Yang, Y.; Chen, X.; Liu, J.; Chen, C.; Yuan, S.; Yu, L. Chin. Chem. Lett. 2020, 31, 1887.

(b) Liu, Y.; Ling, H.; Chen, C.; Xu, Q.; Yu, L.; Jiang, X. Synlett 2019, 30, 1698 .

(c) Cao, H.; Liu, M.; Qian, R.; Zhang, X.; Yu, L. Appl. Organomet. Chem. 2019, 33, e4599.

(d) Jing, X.; Chen, C.; Deng, X.; Zhang, X.; Wei, D.; Yu, L. Appl. Organomet. Chem. 2018, 32, e4332.

(e) Liu, M.; Li, Y.; Yu, L.; Xu, Q.; Jiang, X. Sci. China: Chem. 2018, 61, 294.

[4] Chen, C.; Cao, Y.; Wu, X.; Cai, Y.; Liu, J.; Xu, L.; Ding, K.; Yu, L. Chin. Chem. Lett. 2020, 31, 1078.

[5] (a) Engman, L.; Cava, M. P. Tetrahedron Lett. 1981, 22, 5251.

(b) Kambe, N.; Hondo, K.; Ishii, H.; Sonoda, N. Bull. Chem. Soc. Jpn. 1981, 54, 1460.

(c) Engman, L.; Cava, M. P. J. Org. Chem. 1982, 47, 3946.

[6] (a) Kumar, S.; Engman, L.; Valgimigli, L.; Amorati, R.; Fumo, M. G.; Pedulli, G. F. J. Org. Chem. 2007, 72, 6046.

(b) Kumar, S.; Johansson, H.; Kanda, T.; Engman, L.; Muller, T.; Bergenudd, H.; Jonsson, M.; Pedulli, G. F.; Amorati, R.; Valgimigli, L. J. Org. Chem. 2010, 75, 716.

[7] (a) Liu, C.; Mao, J.; Zhang, X.; Yu, L. Catal. Commun. 2020, 133, 105828.

(b) Yu, L.; Cao, H.; Zhang, X.; Chen, Y.; Yu, L. Sustainable Energy Fuels 2020, 4, 730.

(c) Gao, G.; Han, J.; Yu, L.; Xu, Q. Synlett 2019, 30, 1703.

(d) Yang, Y.; Fan, X.; Cao, H.; Chu, S.; Zhang, X.; Xu, Q.; Yu, L. Catal. Sci. Technol. 2018, 8, 5017.

(e) Wang, T.; Jing, X.; Chen, C.; Yu, L. J. Org. Chem. 2017, 82, 9342.

[8] Trofimov, B. A.; Gusarova, N. K.; Tatarinova, A. A.; Potapov, V. A.; Sinegovskaya, L. M.; Amosova, S. V.; Voronkov, M. G. Tetrahedron 1988, 44, 6739 .

[9] Han, L.; Mirzaei, F.; Tanaka, M. Organometallics 2000, 19, 722.

[10] Jiang, M.; Guo, J.; Liu, B.; Tan, Q.; Xu, B. Org. Lett. 2019, 21, 8328.

[11] Bergman, J.; Engman, L. J. Org. Chem. 1982, 47, 5191.

[12] Fujiwara, S.; Tsutomu, S. Tetrahedron Lett. 1992, 33, 7021.

[13] Barros, S. M.; Dabdoub, M. J.; Dabdoub, V. M. B.; Comasseto, J. V. Organometallics 1989, 8, 1661.

[14] Ogawa, A.; Yokoyama, K.; Obayashi, R.; Han, L.; Kambe, N.; Sonoda, N. Tetrahedron 1993, 49, 1177.

[15] Kumar, S.; Engman, L. J. Org. Chem. 2006, 71, 5400.

[16] Higgs, D. E.; Nelen, M. I.; Detty, M. R. Org. Lett. 2001, 3, 349.

[17] Back, T. G.; Kuzma, D.; Parvez, M. J. Org. Chem. 2005, 70, 9230.

[18] (a) Yu, L.; Li, H.; Zhang, X.; Ye, J.; Liu, J.; Xu, Q.; Lautens, M. Org. Lett. 2014, 16, 1346.

(b) Zhang, X.; Sun, J.; Ding, Y.; Yu, L. Org. Lett. 2015, 17, 5840.

(c) Chen, R.; Qi, J.; Mao, Z.; Cui, S. Org. Biomol. Chem. 2016, 14, 6201 .

(d) Wang, Y.; Wu, Z.; Li, Q.; Zhu, B.; Yu, L. Catal. Sci. Technol. 2017, 7, 3747 . (e) Jing, X.; Wang, T.; Ding, Y.; Yu, L. Appl. Catal. A-Gen. 2017, $541,107$.

(f) Zhang, D.; Huang, Y.; Zhang, E.; Yi, R.; Chen, C.; Yu, L.; Xu, Q. Adv. Synth. Catal. 2018, 360, 784 .

(g) Chu, S.; Cao, H.; Chen, T.; Shi, Y.; Yu, L. Catal. Commun. 2019, 129, 105730

[19] (a) Corey, E. J.; Hopkins, P. B.; Kim, S.; Yoo, S. E.; Nambiar, K. P.; Falck, J. R. J. Am. Chem. Soc. 1979, 101, 7131.

(b) Grirrane, A.; Corma, A.; Garcia, H. J. Catal. 2009, 268, 350.

(c) Royals, E. E.; Horne Jr., S. E. J. Am. Chem. Soc. 1951, 73, 5856.

(d) Zheng, Y.; Wu, A.; Ke, Y.; Cao, H.; Yu, L. Chin. Chem. Lett. 2019, 30, 937 .

[20] Deng, X.; Chao, H.; Chen, C.; Zhou, H.; Yu, L. Sci. Bull. 2019, 64, 1280 .

[21] (a) Jing, X.; Yuan, D.; Yu, L. Adv. Synth. Catal. 2017, 359, 1194. (b) Chen, C.; Zhang, X.; Cao, H.; Wang, F.; Yu, L.; Xu, Q. Adv. Synth. Catal. 2019, 361, 603.

[22] (a) Ou, W.; Zou, R.; Han, M.; Yu, L.; Su, C. Chin. Chem. Lett. 2020, 31, 1899.

(b) Cao, H.; Qian, R.; Yu, L. Catal. Sci. Technol. 2020, 10, 3113.

(c) Bao, W.; He, M.; Wang, J.; Peng, X.; Sung, M.; Tang, Z.; Jiang, S.; Cao, Z.; He, W. J. Org. Chem. 2019, 84, 6065.

(d) Cao, Z.; Zhu, Q.; Lin, Y.; He, W. Chin. Chem. Lett. 2019, 30, 2132 .

(e) Zhang, H.; Han, M.; Yang, C.; Yu, L.; Xu, Q. Chin. Chem. Lett. 2019, 30, 263.

(f) Yu, L.; Qian, R.; Deng, X.; Wang, F.; Xu, Q. Sci. Bull. 2018, 63, 1010 .

(g) Cao, H.; Zhu, B.; Yang, Y.; Xu, L.; Yu, L.; Xu, Q. Chin. J. Catal. 2018, 39, 899 .

(h) Fan, X.; Yi, R.; Wang, F.; Zhang, X.; Xu, Q.; Yu, L. Chin. J. Org. Chem. 2018, 38, 2736 (in Chinese).

(范昕，易容，王芳，张旭，徐清，俞否，有机化学，2018，38, 2736.)

(i) Yu, L.; Wang, J.; Cao, H.; Ding, K.; Xu, Q. Chin. J. Org. Chem. 2014, 34, 1986 (in Chinese).

(俞磊, 王俊, 曹洪恩, 丁克鸿, 徐清, 有机化学, 2014, 34, 1986.)

[23] (a) Tong, Q.; Zhao, S.; Liu, Y.; Xu, B.; Yu, L.; Fan, Y. Appl. Organomet. Chem. 2020, 34, e5380.

(b) Zhao, S.; Xu, B.; Yu, L.; Fan, Y. Chin. Chem. Lett. 2018, 29, 884.

(c) Zhao, S.; Xu, B.; Yu, L.; Fan, Y. Chin. Chem. Lett. 2018, 29 475 .

(d) Wang, F.; Xu, L.; Sun, C.; Yu, L.; Xu, Q. Appl. Organomet. Chem. 2018, 32, e4505.

[24] (a) Zhu, Z.; Wang, W.; Zeng, L.; Zhang, F.; Liu, J. Catal. Commun. 2020, 142, 106031 .

(b) Yang, Y.; Xu, B.; He, J.; Shi, J.; Yu, L.; Fan, Y. Appl. Organomet. Chem. 2019, 33, e5204.

(c) Yang, Y.; Li, M.; Cao, H.; Zhang, X.; Yu, L. Mol. Catal. 2019, 474, 110450

(d) Fan, X.; Yao, Y.; Xu, Y.; Yu, L.; Qiu, C. ChemCatChem 2019, 11, 2596.

(e) Wang, Q.; Jing, X.; Han, J.; Yu, L.; Xu, Q. Mater. Lett. 2018, 215,65 .

(f) Zhang, D.; Wei, Z.; Yu, L. Sci. Bull. 2017, 62, 1325.

[25] (a) Liu, Z.; Zha, M.; Wang, Q.; Hu, G.; Feng, L. Chem. Commun. 2020, 56, 2352.

(b) Zhang, D.; Deng, X.; Zhang, Q.; Han, J.; Yu, L. Mater. Lett. 2019, 234, 216.

[26] Cao, X.; Chen, S.; Bao, M.; Shi, H.; Li, W. Prog. Chem. 2018, 30, 1380 (in Chinese).

(曹小卫, 陈帅, 鲍敏, 史宏灿, 李巍, 化学进展, 2018, 30, 1380.) 